\title{
A rare case of subcutaneous Sweet's syndrome in a patient with chronic myelogenous leukemia: a case report and review of the literature
}

\author{
Congli Wang • Mary Ellen Martin • Roberta E. Smith • \\ Deline DaCosta • Raghava Levaka Veera • Irma E. Palazzo
}

Received: 18 February 2014 / Accepted: 23 May 2014 /Published online: 1 June 2014

(C) Springer-Verlag Berlin Heidelberg 2014

\begin{abstract}
Subcutaneous Sweet's syndrome (SS) is a rare variant of classic SS characterized by a neutrophilic infiltrate exclusively or predominantly in the subcutaneous tissue, with minimal or absent dermal involvement. We report the case of a patient with a history of chronic myelogenous leukemia who developed subcutaneous SS. Although it has been described in patients with myelodysplastic syndromes and acute myeloid leukemia, this is the first report, to our knowledge, of this rare entity occurring in the setting of a myeloproliferative disorder.
\end{abstract}

Keywords Subcutaneous Sweet's syndrome $\cdot$ Chronic myelogenous leukemia $\cdot$ Neutrophilic infiltrates $\cdot$ Myeloid disorders

C. Wang

Department of Pathology \& Laboratory Medicine, Temple

University Hospital, Philadelphia, PA 19140, USA

\section{E. Martin}

Department of Medical Oncology, Fox Chase Cancer Center,

Philadelphia, PA 19111, USA

R. E. Smith • D. DaCosta $・$ I. E. Palazzo

Department of Pathology, Jeanes Hospital, Philadelphia, PA 19111, USA

R. Levaka Veera

Department of Hematology/Oncology, Temple University Hospital/

Fox Chase Cancer Center, Philadelphia, PA 19111, USA

C. Wang $(\bowtie)$

Department of Pathology and Laboratory Medicine, Temple

University School of Medicine, 3401 North Broad Street,

Philadelphia, PA 19140, USA

e-mail: congli.wang@tuhs.temple.edu

\section{Introduction}

Sweet's syndrome (SS), also known as acute febrile neutrophilic dermatosis, is characterized by the sudden onset of fever, multiple tender erythematous papules and plaques, and the presence of intense neutrophilic infiltrates on skin biopsy that are typically located in the upper dermis. SS is often associated with hematologic disease, and to a lesser extent with solid tumors [1-5].

Subcutaneous SS is a rare variant in which the neutrophilic infiltrate is exclusively or predominantly located in the subcutaneous tissue, with minimal or no dermal involvement [6, 7]. Subcutaneous SS is almost exclusively associated with myeloid disorders. To the best of our knowledge, only 16 cases have been reported in such a setting in the Englishlanguage literature to date [8-19], including ten cases with myelodysplastic syndromes (MDS) and six cases with acute myeloid leukemia (AML). We describe the case of a patient with chronic myelogenous leukemia (CML) who developed subcutaneous SS, an association which has not been previously reported.

\section{Case report}

The patient is a 43-year-old black female from Bermuda with a history of CML admitted for anemia, thrombocytopenia, fever, fatigue, splenomegaly and abdominal pain, and severe right leg pain. She was diagnosed with CML that was confirmed by the expression of BCR-ABL by FISH, in January 2009. She failed therapy with multiple tyrosine kinase inhibitors including ponatinib. At the time of hospital admission, her hemoglobin was $4 \mathrm{~g} / \mathrm{dl}$, platelet count was 5,000/ul, and the white blood cell count was $2,500 /$ ul with $9 \%$ circulating blasts. She received two units of packed red blood cells and two units of platelets. Plain films and MRI of the right hip 
joint excluded a pathological fracture. Additionally, Cefepime was started for fever. On physical examination at the time of admission, two firm, tender subcutaneous nodules were noted on the medial side of the right thigh measuring $4 \times 4$ and $3 \times$ $2 \mathrm{~cm}$, respectively. Within $24 \mathrm{~h}$ of admission, she developed new left arm pain and worsening abdominal pain. A third nodule developed on the left arm measuring $2 \times 2 \mathrm{~cm}$ (Fig. 1). The overlying skin appeared erythematous without fluctuance or pustule. CT scan of the chest, abdomen, and pelvis with contrast showed multiple nodular densities in both lungs as well as hepatosplenomegaly. Micafungin was added for suspected disseminated fungal infection. On day 5 of her hospitalization, two more firm, tender, erythematous nodules were noted on the right arm and forearm. Given the concern for disease evolution and for leukemia cutis, biopsies of the bone marrow and one of the subcutaneous nodules were performed.

Bone marrow biopsy revealed a hypercellular marrow with prominent myeloid hyperplasia, left-shifted granulopoiesis, and markedly decreased megakaryocytic and erythroid precursors. CD34 positive cells comprised $5 \%$ of the marrow. The peripheral blood smear revealed $8 \%$ blasts and $67 \%$ segmented neutrophils. Biopsy of the subcutaneous nodule showed organizing fat necrosis and a dense neutrophilic infiltrate in the lobules, with associated fibrinoid necrosis and numerous macrophages (Fig. 2). There was no significant dermal involvement and no evidence of vasculitis. In addition, no blasts were seen; this was confirmed by the absence of staining for CD34 on immunohistochemistry. Special stains for fungus, acid-fast bacilli, and bacteria were all negative. A diagnosis of subcutaneous SS was made.

During her hospitalization, the percentage of peripheral circulating blasts rose to $18 \%$. With a concern for disease progression, induction chemotherapy was started with cytarabine and idarubicin $(7+3$ regimen). The patient had a significant clinical improvement, and she is currently awaiting

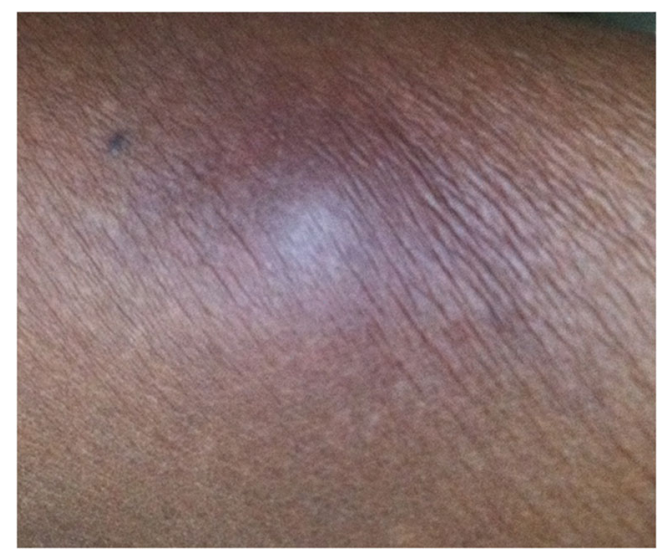

Fig. 1 Subcutaneous firm, erythematous nodule on the left arm. Similar nodules were present on the right thigh and right arm (not shown)

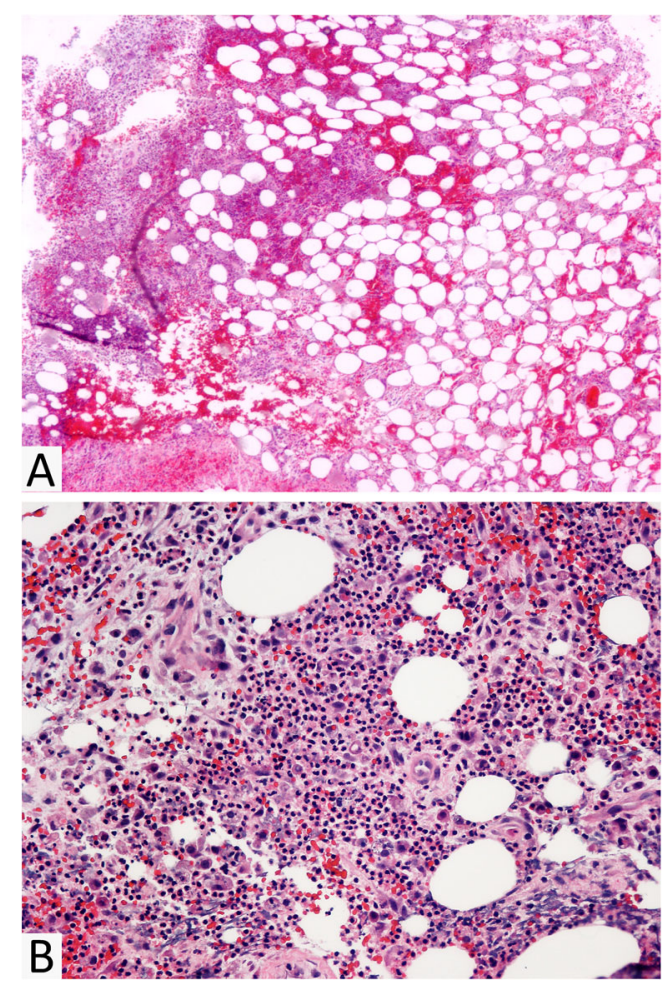

Fig. 2 Histological examination showing organizing fat necrosis and dense lobular neutrophilic infiltrate, with associated fibrinoid necrosis and numerous macrophages (hematoxylin-eosin stain; original magnifications: $\mathbf{a} \times 20, \mathbf{b} \times 200$ )

haploidentical hematopoietic stem cell transplantation. Interestingly, she did not receive steroids, and her nodules responded to chemotherapy. The two nodules on her right arm completely disappeared within a week, and the rest of the nodules decreased in size and became non-tender.

\section{Discussion}

Subcutaneous SS is an extremely rare entity. Although it has been described to occur after treatment with vemurafenib, a BRAF inhibitor [20], the majority of reported cases have been associated with underlying myeloid disorders. Following a comprehensive literature search, we identified 16 cases of subcutaneous SS that developed in such a clinical setting. The clinical and histopathologic features of those lesions are tabulated in Table 1.

Among the 16 previously reported cases, different terms have been used, including neutrophilic panniculitis [11-13, 16, 18], SS-like (Sweet's-like) neutrophilic panniculitis [14], and Sweet's panniculitis [10]. In all the cases, the patients had either MDS or AML and presented with multiple erythematous nodules that frequently involved the limbs, especially the lower extremities [8-19]. Most of the nodules were accompanied by fever, and some were painful. On histological examination, all lesions showed an extensive neutrophilic infiltrate 


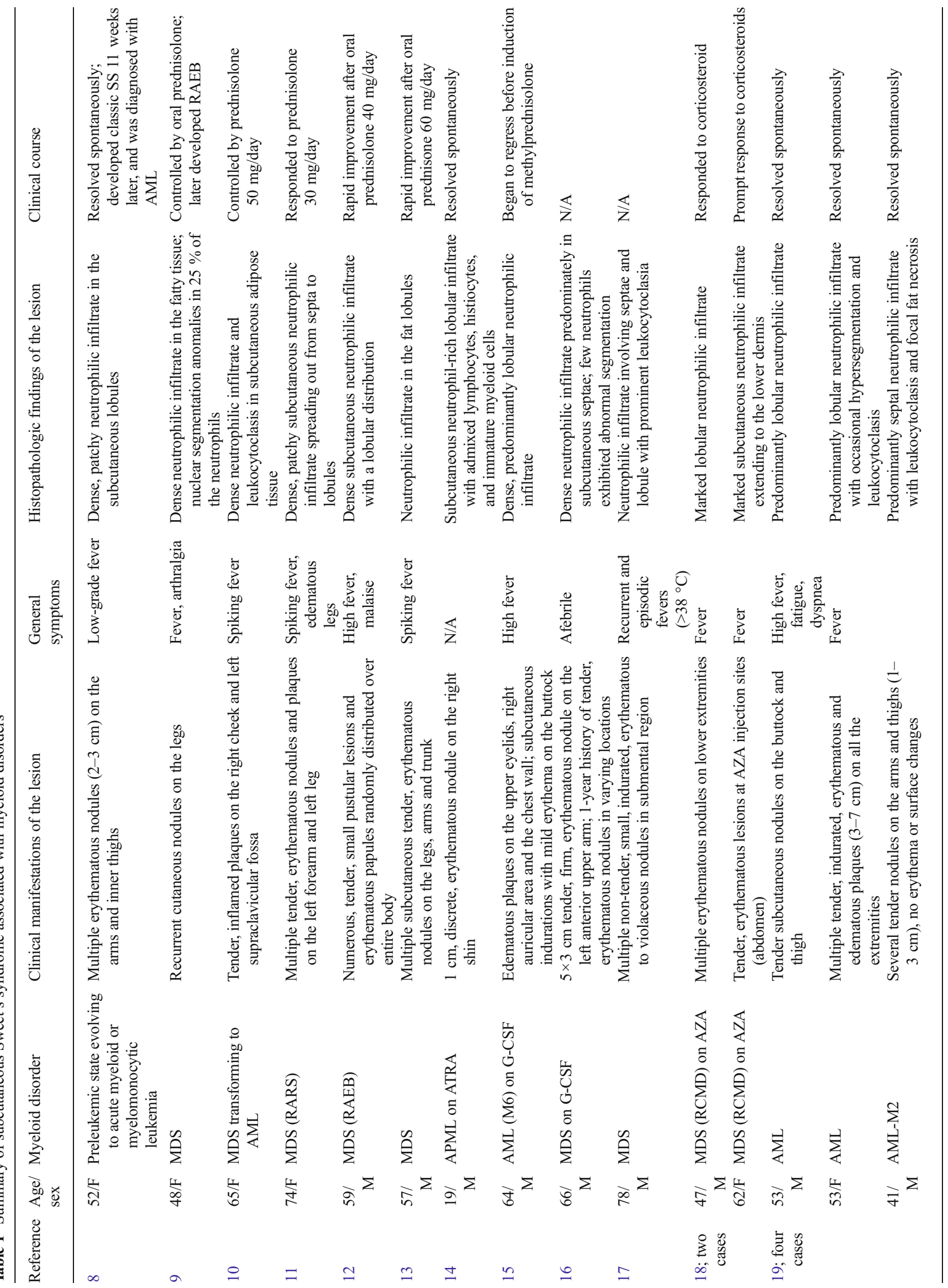




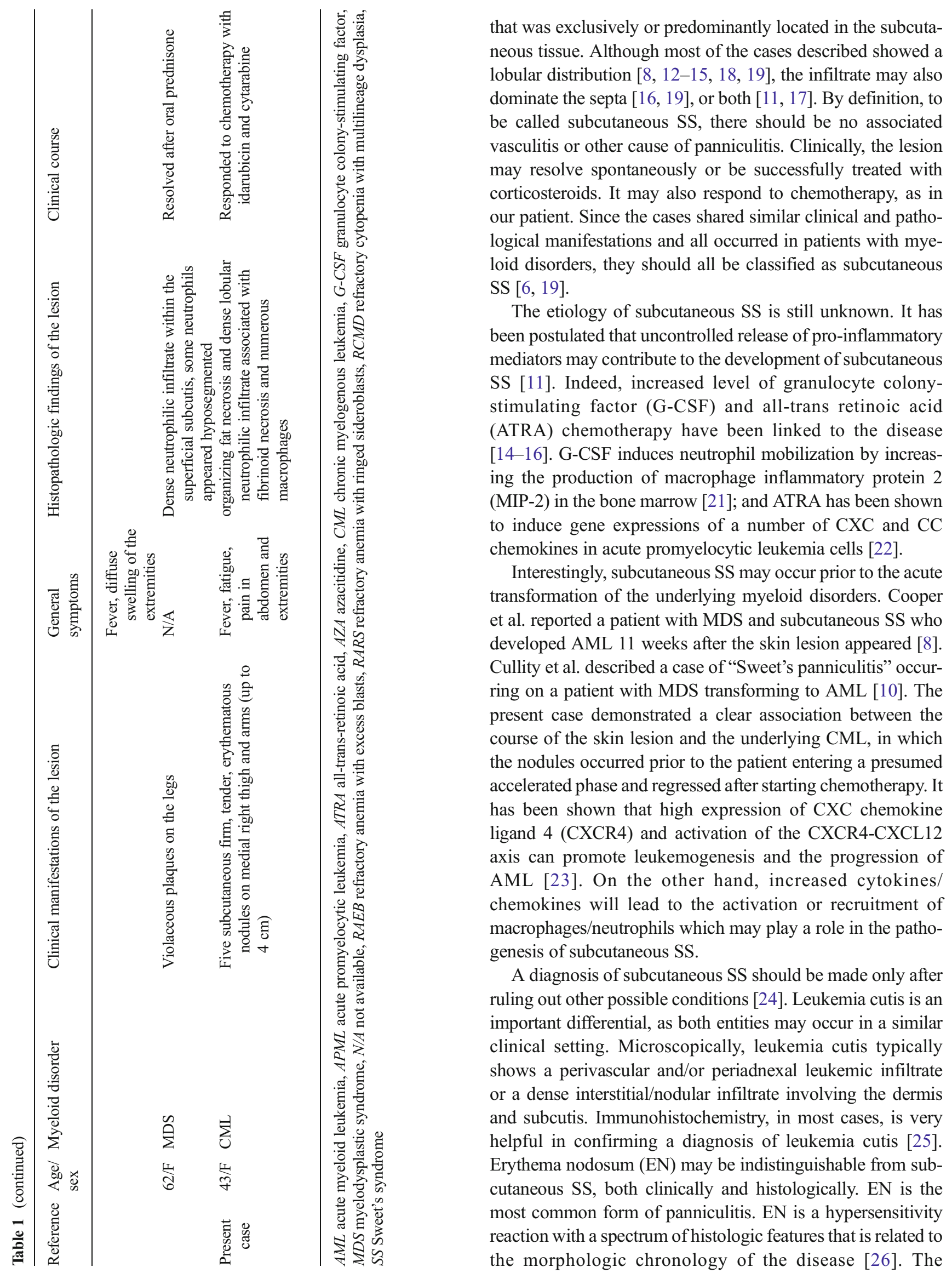


prototypical lesions present as a septal panniculitis with a mixed cellular infiltrate comprising lymphocytes, neutrophils, histiocytes, giant cells, and variable eosinophils. Occasionally, EN may demonstrate a predominant neutrophilic component [27]. The presence of Miescher radial granulomas is thought to be relatively specific for EN [7, 19]. However, it has also been reported in SS, Behcet's disease, and necrobiosis lipoidica [28]. Additionally, several other conditions that can lead to subcutaneous neutrophilic inflammation should be excluded, including infection, pancreatic panniculitis, alpha1 antitrypsin deficiency, rheumatoid arthritis, with inflammatory bowel disease, and local reaction secondary to injection $[6,19,24]$.

In conclusion, we report the first case of subcutaneous SS occurring in the setting of CML. Subcutaneous SS is a rare entity associated with MDS, AML, and CML. In our opinion, it is important to recognize and differentiate subcutaneous SS from other diseases and conditions, as subcutaneous SS may be a warning sign of progression of the underlying myeloid disorder.

Conflict of interest The authors declare that they have no conflict of interest.

\section{References}

1. Callen JP (2002) Neutrophilic dermatoses. Dermatol Clin 20:409419

2. Clemmensen OJ, Menné T, Brandrup F, Thomsen K, Lange Wantzin G (1989) Acute febrile neutrophilic dermatosis - a marker of malignancy. Acta Derm Venereol 69:52-58

3. Buck T, Gonzalez LM, Lambert WC, Schwartz RA (2008) Sweet's syndrome with hematologic disorders: a review and reappraisal. Int J Dermatol 47:775-782

4. Cohen PR, Holder WR, Tucker SB, Kono S, Kurzrock R (1993) Sweet's syndrome in patients with solid tumors. Cancer 72:27232731

5. von den Dreisch P (1994) Sweet's syndrome (acute febrile neutrophilic dermatosis). J Am Acad Dermatol 31:535-556

6. Cohen PR (2005) Subcutaneous Sweet's syndrome: a variant of acute febrile neutrophilic dermatosis that is included in the histopathologic differential diagnosis of neutrophilic panniculitis. J Am Acad Dermatol 52(5):927-928

7. Guhl G, García-Díez A (2008) Subcutaneous sweet syndrome. Dermatol Clin 26(4):541-551

8. Cooper PH, Frierson HF, Greer KE (1983) Subcutaneous neutrophilic infiltrates in acute febrile neutrophilic dermatosis. Arch Dermatol 119:610-611

9. Morioka M, Otsuka F, Nogita T, Igisu K, Urabe A, Ishibashi Y (1990) Neutrophilic dermatosis with myelodysplastic syndrome: nuclear segmentation anomalies of neutrophils in the skin lesion and in peripheral blood. J Am Acad Dermatol 23:247-249

10. Cullity J, Maguire B, Gebauer K (1991) Sweet's panniculitis. Australas J Dermatol 32:61-64

11. Matsumura Y, Tanabe H, Wada Y, Ohta K, Okamoto H, Imamura S (1997) Neutrophilic panniculitis associated with myelodysplastic syndromes. Br J Dermatol 136:142-144

12. Chen HC, Kao WY, Chang DM, Gao HW, Lai WY, Lai JH (2004) Neutrophilic panniculitis with myelodysplastic syndromes presenting as pustulosis: case report and review of the literature. Am J Hematol 76:61-65

13. Sutra-Loubet C, Carlotti A, Guillemette J, Wallach D (2004) Neutrophilic panniculitis. J Am Acad Dermatol 50:280-285

14. Jagdeo J, Campbell R, Long T, Muglia J, Telang G, RobinsonBostom L (2007) Sweet's syndrome-like neutrophilic lobular panniculitis associated with all-trans-retinoic acid chemotherapy in a patient with acute promyelocytic leukemia. J Am Acad Dermatol 56:690-693

15. Uhara H, Saida T, Nakazawa H, Ito T (2008) Neutrophilic dermatoses with acute myeloid leukemia associated with an increase of serum colony-stimulating factor. J Am Acad Dermatol 59(Suppl):S10-S12

16. Becherer K, Golda N, Feldman M, Diaz-Arias A, Caldwell C (2009) Neutrophilic panniculitis associated with myelodysplastic syndrome with abnormal nuclear forms. J Cutan Pathol 36:1024-1026

17. Hood M, Yu K, Magro C, Reisacher W (2010) Pathology quiz case 2: subcutaneous sweet syndrome of the neck. Arch Otolaryngol Head Neck Surg 136(1038):1040-1041

18. Kim IH, Youn JH, Shin SH, Yahng SA, Lee SE, Kwon JC, Lee DG, Park KS, Choi MH, Jung SE, Kim YJ (2012) Neutrophilic panniculitis following azacitidine treatment for myelodysplastic syndromes. Leuk Res 36:e146-e148

19. Chan MP, Duncan LM, Nazarian RM (2013) Subcutaneous sweet syndrome in the setting of myeloid disorders: a case series and review of the literature. J Am Acad Dermatol 68(6):1006-1015

20. Kim GH, Levy A, Compoginis G (2013) Neutrophilic panniculitis developing after treatment of metastatic melanoma with vemurafenib. J Cutan Pathol 40(7):667-669

21. Nguyen-Jackson HT, Li HS, Zhang H, Ohashi E, Watowich SS (2012) G-CSF-activated STAT3 enhances production of the chemokine MIP-2 in bone marrow neutrophils. J Leukoc Biol 92(6):12151225

22. Shibakura M, Niiya K, Niiya M, Asaumi N, Yoshida C, Nakata Y, Tanimoto M (2005) G-CSF-activated STAT3 enhances production of the chemokine MIP-2 in bone marrow neutrophils. Leuk Res 29(7): 755-759

23. Ayala F, Dewar R, Kieran M, Kalluri R (2009) Contribution of bone microenvironment to leukemogenesis and leukemia progression. Leukemia 23:2233-2241

24. Gerami P, Guitart J (2007) Panniculitis with histiocytoid/immature neutrophils is not limited to histiocytoid panniculitic Sweet syndrome. Am J Clin Pathol 29(6):596

25. Cho-Vega JH, Medeiros LJ, Prieto VG, Vega F (2008) Leukemia cutis. Am J Clin Pathol 129(1):130-142

26. White WL, Hitchcock MG (1999) Diagnosis: erythema nodosum or not? Semin Cutan Med Surg 18:47-55

27. Thurber S, Kohler S (2006) Histopathologic spectrum of erythema nodosum. J Cutan Pathol 33:18-26

28. White WL, Wieselthier JS, Hitchcock MG (1996) Panniculitis: recent developments and observations. Semin Cutan Med Surg 15:278-299 